\title{
On a Class of Relativistic Invariant Distributions
}

\author{
G. V. Efimov \\ Joint Institute for Nuclear Research \\ Laboratory of Theoretical Physics \\ Received July 12, 1967
}

\begin{abstract}
Space-time properties of a class of relativistic invariant distributions of the type

$$
K\left(x-x^{\prime}\right)=\sum_{n=0}^{\infty} \frac{c_{n}}{(2 n) !} \square^{n} \delta^{(4)}\left(x-x^{\prime}\right)
$$

were considered for different sequences of coefficients $c_{n}$.
\end{abstract}

\section{Introduction}

The assumption of a character of distributions takes essential place among the basis postulates of the local quantum field theory. Such distributions are coefficient functions in the expansion of the S-matrix in a series of field operator normal products [1] or are Wightman's functions in his axiomatic approach [2]. This assumption is connected with a definition of local properties of distributions. The important class of the tempered distributions, becoming a traditional one in investigation of the quantum field theory, seems to be chosen because the concept of locality is introduced here by the most simple and natural manner. But lately it has become more clear that this class is not always an adequate instrument. It turns out that the class of local distributions can be extended essentially. The most significant results were received by MEIMAN [3] and JAFFE [4]. It is remarkable that the definition of concept of microcausality by MEIMAN and strict localizability by JAFFE takes the basic place in the papers of these authors. They received the different classes of the test functions depending on the definitions introduced. MAIMAN's and JAFFE's idea is to choose the "minimum" class of test functions. It means the following. If one introduces a certain definition of locality then only such distributions must be defined on the "minimum" class of test functions which satisfy the definition introduced. The other distributions must not be defined on the whole class of test functions. The requirement of the "minimality" permits to get the important physical consequences from this purely mathematical hypothesis following from the definition of locality only. (For example, CPT-theorem, theorem of local commutativity, restriction on behaviour of amplitudes when energy goes to infinity and so on.) 
The choice of the „,minimum" class of test functions is justified completely if we hold the axiomatic approach in the quantum field theory and want to deduce physical consequences from the most general requirements. If we hold the dynamical picture in the quantum field theory (for example, the construction of the perturbation series for the S-matrix according to the interaction Lagrangian), then the dynamical description of a interaction itself should dictate which class of test functions and distributions should be chosen for a correct description of the interaction picture. It may occur that the interaction is not local. But at the present time we have no good definition of a relativistic invariant non-local coupling between quantum field operators. We try to give such definition in this paper. With this aim we will study the space-time properties of relativistic invariant distributions of the type

where

$$
K\left(x-x^{\prime}\right)=\sum_{n=0}^{\infty} \frac{c_{n}}{(2 n) !} \square^{n} \delta^{(4)}\left(x-x^{\prime}\right)
$$

$$
\square=-\frac{\partial^{2}}{\partial x_{0}^{2}}+\frac{\partial^{2}}{\partial x_{1}^{2}}+\frac{\partial^{2}}{\partial x_{2}^{2}}+\frac{\partial^{2}}{\partial x_{3}^{2}}
$$

for different sequences of coefficients $c_{n}$. We will destinguish the following possiblities

$$
\begin{aligned}
& \varlimsup_{n \rightarrow \infty}\left|c_{n}\right|^{\frac{1}{n}}=0 \\
& \varlimsup_{n \rightarrow \infty}\left|c_{n}\right|^{\frac{1}{n}}=l^{2}<\infty \\
& \varlimsup_{n \rightarrow \infty}\left|c_{n}\right|^{\frac{1}{n}}=\infty, \quad \varlimsup_{n \rightarrow \infty}\left|\frac{c_{n}}{(2 n) !}\right|^{\frac{1}{n}}=0, \\
& \varlimsup_{n \rightarrow \infty}\left|\frac{c_{n}}{(2 n) !}\right|^{\frac{1}{n}}=a^{2}<\infty .
\end{aligned}
$$

In $\S 2$ we describe the class of test functions and give the definition of support of distributions, in $\S \S 3-5$ we consider distributions fo the type (1.1) for sequences $c_{n}$ satisfying the conditions $(1.2-5)$.

\section{The Class of Test Functions}

We choose the space 3 of all entire analytic functions $f(z) \equiv$ $\equiv f\left(z_{0}, z_{1}, z_{2}, z_{3}\right) \equiv f\left(z_{0}, \mathbf{z}\right)$ depending on four variables $z_{j}=x_{j}+i y_{j}$ $(j=0,1,2,3)$ in the capacity of the class of our test functions. We define in space 3 the countable sequence of the norms

$$
\|f\|_{m}=\max _{\left|z_{j}\right|<m}\left|f\left(z_{0}, z_{1}, z_{2}, z_{3}\right)\right| \text {. }
$$

We will say that the sequence of functions $\left\{f_{K}(z)\right\}$ where $f_{K}(z) \in \mathcal{B}$ converges correctly if it converges uniformly in any bounded region of real values of arguments $\left(x_{0}, x_{1}, x_{2}, x_{3}\right)$. 
Lemma. If each function of the sequence $\left\{f_{K}(z)\right\}$, where $f_{K}(z) \in B$, is bounded according to norm $\left\|f_{K}\right\|_{m}$ by the constant $M$ and this sequence converges correctly, then its limit $f(z)$ belongs to the space 8 and the norm of $f(z)$ does not surpass $M$.

This lemma is the other formulation of Vitaly's theorem about convergence of analytic functions sequences [5]. Therefore the norms introduced are compatible and the space $\mathcal{B}$ is complete and perfect.

We give one more

Definition. The sequence $\left\{f_{K}(z)\right\}$, where $f_{K}(z) \in \mathcal{B}$, converges in the region $G \subset C^{4}$ if it converges uniformly in the closed region $G$.

The space $\mathcal{B}$ is not closed relative to this convergence definion. But we will make use of it for another definition of the support of a functional on the space 8 .

The space of all linear continuous functionals defined on the space 8 is denoted by $\boldsymbol{B}^{\prime}$. The explicit form of such functionals can be found according to usual procedure $[6,7]$.

$$
(F, f)=\int_{\left|z_{j}\right| \leqq m} f(z) d \mu_{F}(z)
$$

where $\mu_{F}(z)$ is a complex completely additive measure in the region $\left|z_{j}\right| \leqq m$. This formula with all possible $m$ gives the general form of linear continuous functionals on the space $\mathcal{B}$.

Let us introduce the concept of the functional support. We give the following

Definition. The region $G \subset C^{4}$ is said to be the support of the functional $F \in \mathcal{B}^{\prime}$ if this functional can be extended continuously on the space $\mathcal{B}(G)$ of functions, which are analytical in the closed region $G$.

If the region $G$ is a point $z=\left(z_{0}, z_{1}, z_{2}, z_{3}\right)$ then functional is said to be local.

It follows from given definitions that the sequence of numbers $\left(F, f_{K}\right)$ converges to zero if the support of the functional $F$ is a region $G$ and $\left\{f_{K}\right\}$ where $f_{K}(z) \in \mathcal{B}$ is any sequence which converges to zero in $G$.

\section{The Local Distributions}

Let us consider the distributions (1.1) where the coefficians $c_{n}$ satisfy the condition (1.2) We consider the functional:

$$
(K, f)(x)=\int d^{4} x^{\prime} K\left(x-x^{\prime}\right) f\left(x^{\prime}\right)=\sum_{n=0}^{\infty} \frac{c_{n}}{(2 n) !} \square^{n} f(x) .
$$

We make use of the following integral representation for the operator $\square$ :

$$
\begin{aligned}
\square^{n} & =\left(-\frac{\partial^{2}}{\partial x_{0}^{2}}+\frac{\partial^{2}}{\partial \boldsymbol{x}^{2}}\right)^{n}=a_{n} \int_{\varrho^{2} \leqq 1} d^{4} \varrho\left(i \varrho_{4} \frac{\partial}{\partial x_{0}}+\varrho \frac{\partial}{\partial \boldsymbol{x}}\right)^{2 n} \\
a_{n} & =\frac{2^{2 n}}{\pi^{2}} \cdot \frac{n !(n+2) !}{(2 n) !} ; \lim _{n \rightarrow \infty}\left|a_{n}\right|^{\frac{1}{n}}=1 .
\end{aligned}
$$


The integration in (3.2) is performed over the Euclidean globe

$$
\varrho^{2}=\varrho_{1}^{2}+\varrho_{2}^{2}+\varrho_{3}^{2}+\varrho_{4}^{2}=\varrho^{2}+\varrho_{4}^{2} \leqq 1 \text {. }
$$

Let us introduce the function of a complex variable $\xi$ :

$$
W(\xi)=\sum_{n=0}^{\infty} \frac{c_{n} a_{n}}{\xi^{2 n+1}}
$$

It follows from the conditions (1.2) and (3.3) that $W(\xi)$ has the only essential singularity at $\xi=0$ and has not other singularities in the complex plante of $\xi$. Therefore

$$
c_{n} a_{n}=\frac{1}{2 \pi i} \oint d \xi \xi^{2 n} W(\xi)
$$

where the integration is performed over any closed contour around the point $\xi=0$. Making use of (3.2) and (3.5) the functional (3.1) can be written in the form

$$
\begin{aligned}
(K, f)(x) & =\sum_{n=0}^{\infty} \frac{1}{(2 n) !} \cdot \frac{1}{2 \pi i} \oint d \xi \xi^{2 n} W(\xi) \int_{\varrho^{2} \leqq 1} d^{4} \varrho\left(i \varrho_{4} \frac{\partial}{\partial x_{0}}+\varrho \frac{\partial}{\partial \boldsymbol{x}}\right)^{2 n} f(x) \\
& =\frac{1}{2 \pi i} \oint d \xi W(\xi) \int_{\varrho^{2} \leqq 1} d^{4} \varrho \sum_{n=0}^{\infty} \frac{\xi^{n}}{n !}\left(i \varrho_{4} \frac{\partial}{\partial x_{0}}+\varrho \frac{\partial}{\partial \boldsymbol{x}}\right)^{n} f\left(x_{0}, \boldsymbol{x}\right) .
\end{aligned}
$$

In the last term the summation is performed over all $n$ because the integral over $\varrho$ of the odd powers of $\left(i \varrho_{4} \frac{\partial}{\partial x_{0}}+\varrho \frac{\partial}{\partial \boldsymbol{x}}\right)$ is identically equal to zero. Observing that there is the displacement operator with respect to the arguments $\left(x_{0}, \boldsymbol{x}\right)$ in $(3.6)$ one can get

$$
(K, f)(x)=\frac{1}{2 \pi i} \oint d \xi W(\xi) \int_{\varrho^{2} \leqq 1} d^{4} \varrho f\left(x_{0}+i \varrho_{4} \xi, \boldsymbol{x}+\varrho \xi\right) .
$$

This expression is well defined because of $f\left(x_{0}, \boldsymbol{x}\right) \in \mathcal{B}$.

The integration over $\xi$ can be performed over counter with an arbitrary small radius because $W(\xi)$ has the only singularity at the point $\xi=0$. Therefore the functional (3.7) can be extended continuously on the space of functions which are analytic at the point $x=\left(x_{0}, \boldsymbol{x}\right)$. Hence the distributions (1.1) with the condition (1.2) are local according to our definition.

The Fourier-transform of the distributions (1.1) can be written in the form

$$
\widetilde{K}\left(p^{2}\right)=\sum_{n=0}^{\infty} \frac{c_{n}}{(2 n) !}\left(p^{2}\right)^{n}
$$

The function $\widetilde{K}\left(p^{2}\right)$ is an entire analytic function of the order $\gamma<\frac{1}{2}$ in the complex plane $p^{2}$, i.e.

$$
\left|\widetilde{K}\left(p^{2}\right)\right|<e^{\varepsilon \sqrt{\left|p^{2}\right|}}
$$

for any $\varepsilon>0$. According to the theory of entire analytic functions (see e.g. [6]) it is known that for these functions there is not any direction in 10 Commun. math. Phys., Vol. 7 
the complex plane $p^{2}$, along which they could decrease. It means that they cannot play the role of the cutoff functions in the constructing of the perturbation theory for the S-matrix if we somehow want to make use of the quasi-local arbitrariness in constructing the coefficient functions in the perturbation expansion of the S-matrix.

The "minimum" space of test functions in this case is the class $3^{0}$ of functions which are analytic with respect to each argument $z_{j}=x_{j}+i y_{j}$ in some band $\left|y_{j}\right|<d_{j}$ where $d_{j}$ depends on the function $f(z)$ and can be arbitrary quantity. The fixed band $\left|y_{j}\right|<d_{0}$ where all functions $f \in B$ are analytic does not exist. In this way we got the relativistic generalization of Merman's class $C_{0}[3]$.

\section{The Non-local Distributions}

Now let us consider the distributions (1.1) where the coefficients $c_{n}$ satisfy the condition (1.3) where $l$ is some parameter. We consider functional $(K, f)(x)$ for which the formula (3.1) is valid. Let us make use of the integral representation (3.2) and introduce the function $W_{l}(\xi)$ of the complex variable $\xi$ according to (3.4). In this case it follows from the conditions (1.3) and (3.3) that the function $W_{l}(\xi)$ is analytic in the complex plane $\xi$ outside of the circle $|\xi|=l$. Inside of this circle it has some singularities the positions of which depend on an explicit form of the coefficients $c_{n}$. Therefore

$$
c_{n} a_{n}=\frac{1}{2 \pi i} \oint_{|\xi|>l} d \xi \xi^{2 n} W_{l}(\xi) .
$$

Repeating the calculations of the preceding section one can get for the functional (1.1) with the condition (1.3) the following representation

$$
(K, f)(x)=\frac{1}{2 \pi i} \oint_{|\xi|>l} d \xi W_{l}(\xi) \int_{\varrho^{2} \leqq 1} d^{4} \varrho f\left(x_{0}+i \varrho_{4} \xi, \boldsymbol{x}+\varrho \xi\right) .
$$

This expression is well defined on 3 .

Let us demonstrate that the representation (4.2) is relativistic covariant on 3 . We have to prove that the integral in (4.2) over the Euclidean space is covariant under coordinate transformations from the homogeneous Lorentz group, i.e.

$$
\begin{aligned}
& \int d^{4} \varrho f\left(\Lambda_{0 j}\left(x_{j}+\varrho_{j} \xi\right), \Lambda_{\beta j}\left(x_{j}+\varrho_{j} \xi\right)\right) \\
= & \int_{\varrho^{2} \leqq 1} d^{4} \varrho f\left(\Lambda_{0 j} x_{j}+i \varrho_{4} \xi, \Lambda_{\beta j} x_{j}+\varrho_{\beta} \xi\right)
\end{aligned}
$$

where $\Lambda$ is a representation of the homogeneous Lorentz group $L$. The summation is performed over $j=(0,1,2,3)$. Here we introduced the notations $\varrho_{0}=i \varrho_{4}$ and $\beta=(1,2,3)$.

Any $\Lambda \in L$ can be represented in the form $\Lambda=\Lambda_{1} \Lambda_{2} \Lambda_{3}$ where $\Lambda_{1}$ and $\Lambda_{3}$ are usual rotations and $\Lambda_{2}$ is the purely Lorentz transformation 
along three-axis. The integral (4.3) obviously is covariant with respect to rotations in the three-dimensional Euclidean space. Therefore it will be sufficient for us to demonstrate the covariantness of the representation (4.2) under the purely Lorentz transformation $\Lambda_{2}$. Then the relation (4.2) can be written

$$
\begin{aligned}
\int_{\varrho^{2} \leqq 1} d^{4} \varrho f\left(\left(x_{0}+i \varrho_{4} \xi\right) \operatorname{Ch} \vartheta+\left(x_{3}+\varrho_{3} \xi\right) \operatorname{Sh} \vartheta, x_{1}+\varrho_{1} \xi, x_{2}+\right. \\
\left.+\varrho_{2} \xi,\left(x_{0}+i \varrho_{4} \xi\right) \operatorname{Sh} \vartheta+\left(x_{3}+\varrho_{3} \xi\right) \operatorname{Ch} \vartheta\right) \\
=\int d_{\varrho^{2} \leqq 1} d^{4} \varrho f\left(x_{0} \operatorname{Ch} \vartheta+x_{3} \operatorname{Sh} \vartheta+i \varrho_{4} \xi, x_{1}+\varrho_{1} \xi, x_{2}+\right. \\
\left.+\varrho_{2} \xi, x_{0} \operatorname{Sh} \vartheta+x_{3} \operatorname{Ch} \vartheta+\varrho_{3} \xi\right)
\end{aligned}
$$

where th $\vartheta=\frac{v}{c}, l<|\xi|<l+\varepsilon$. Let us go over to the polar coordinates in the plane $\left(\varrho_{0}, \varrho_{3}\right)$ and put $\varrho_{0}=r \operatorname{Cos} \varphi, \varrho_{3}=r \operatorname{Sin} \varphi$. Then the left part of (4.4) can be written as

$$
\begin{gathered}
\int_{0}^{2 \pi} d \varphi \int_{0}^{1} d r \cdot r \iint_{\varrho_{1}^{2}+\varrho_{2}^{2} \leqq 1-r^{2}} d \varrho_{1} d \varrho_{2} f\left(x_{0}^{\prime}+i \xi r \operatorname{Cos}(\varphi+i \vartheta), x_{1}+\right. \\
\left.+\varrho_{1} \xi, x_{2}+\varrho_{2} \xi, x_{3}^{\prime}+\xi r \operatorname{Sin}(\varphi+i \vartheta)\right)
\end{gathered}
$$

where $x_{0}^{\prime}=x_{0} \operatorname{Ch} \vartheta+x_{3} \operatorname{Sh} \vartheta, x_{3}^{\prime}=x_{0} \operatorname{Sh} \vartheta+x_{3} \operatorname{Ch} \vartheta$.

In so far as the function $f\left(z_{0}, z_{1}, z_{2}, z_{3}\right)$ is entire analytic with respect to the arguments $z_{j}$ it will be the entire analytic function with respect to the complex argument $\varphi$. In doing so the integrand in (4.5) is periodic along the real axis in the complex plane $\varphi$ with the period $2 \pi$. Therefore in (4.5) one can displace the integration contour over $\varphi$ from 0 to $2 \pi$ by any purely imaginary number, i.e.

$$
\int_{0}^{2 \pi} d \varphi A(\varphi)=\int_{i \vartheta}^{i \vartheta+2 \pi} d \varphi A(\varphi)=\int_{0}^{2 \pi} d \varphi A(\varphi-i \vartheta)
$$

where $A(\varphi)$ is the integrand in (4.5). Thus the right part of (4.4) is equal to the left part and our assertion is proved.

Now let us go over to the study of space-time properties of the distributions $K\left(x-x^{\prime}\right)$ in (4.2). The functional (4.2) can be extended with the relativistic covariance conservation on the space of functions $B\left(G_{l}(x)\right)$ which are analytic in the open region $G_{l}(x)$ where $z^{\prime} \in G_{l}(x)$ if

$$
\left|\left(z^{\prime}-x\right)^{2}\right| \leqq l^{2}
$$

where $\left(z^{\prime}-x\right)^{2}=\left(z_{0}^{\prime}-x_{0}\right)^{2}-\left(\mathbf{z}^{\prime}-\mathbf{x}\right)^{2}$.

The intersection of the region $G_{l}(x)$ with the real space $R^{4}$ is the hyperboloid

$$
-l^{2} \leqq\left(x^{\prime}{ }_{0}-x_{0}\right)^{2}-\left(\mathbf{x}^{\prime}-\mathbf{x}\right)^{2} \leqq l^{2} .
$$

This region in $R^{4}$ is infinite and its 4 -volume is infinite too.

The functional (4.2) can be extended on the wider class of functions. We have proved that the representation (4.2) can be written on the class 
$3\left(G_{l}(x)\right)$ in the following equivalent forms

$$
(K, f)(x)=\frac{1}{2 \pi i} \oint_{|\xi|>l} d \xi W_{l}(\xi) \int_{\varrho^{2} \leqq 1} d^{4} \varrho f(x+\Lambda \varrho \xi)
$$

where $\Lambda=\Lambda(\vartheta, \varphi, \psi)$ is a representation of the homogeneous Lorentz group, $\vartheta$ is the Lorentz angle, $\varphi$ and $\psi$ are the Euclidean angles. If we choose some definite representation with the fixed parameters $\vartheta, \varphi$ and $\psi$ then for given $\Lambda(\vartheta, \varphi, \psi)$ the functional (4.9) can be extended on the class of functions which are analytic in the region

$$
G_{l}(\vartheta, \varphi, \psi ; x) \subset G_{l}(x) .
$$

The point $z^{\prime}$ belongs to $G_{l}(\vartheta, \varphi, \psi ; x)$ when $\vartheta=\varphi=\psi=0$ if

$$
\left|z^{\prime}{ }_{0}-x_{0}\right|^{2}+\left|\mathbf{z}^{\prime}-\mathbf{x}\right|^{2} \leqq l^{2} \text {. }
$$

In the case of arbitrary values of $\vartheta, \varphi$ and $\psi$ the region $G_{l}(\vartheta, \varphi, \psi ; x)$ can be obtained by the Lorentz transformation from the region (4.11). In other words, $G_{l}(\vartheta, \varphi, \psi ; x)$ is a globe in the case $\vartheta=\varphi=\psi=0$ and is a ellipsoid in the case of arbitrary $\vartheta, \varphi$ and $\psi$ which is located entirely inside of the hyperboloid $G_{l}(4.8)$. Emphasize that the region $G_{l}(\vartheta, \varphi, \psi ; x)$ for each given $\vartheta, \varphi$ and $\psi$ is bounded and the 4-volume of all regions $G_{l}(\vartheta, \varphi, \psi ; x)$ is the same. It is finite and proportional to $l^{4}$.

So, the functional (4.9) is extended for each given representation $\Lambda$ on the space of the test functions $3\left(G_{l}(\vartheta, \varphi, \psi ; x)\right)>\mathcal{B}\left(G_{l}(x)\right)>\mathcal{B}$. Consequently the support of the functional (1.1) with the condition (1.3) is some bounded region $G_{l}(\vartheta, \varphi, \psi ; x) \subset G_{l}(x)$.

The same could be done in a somewhat different way. We could choose a sequence $f_{n}(z) \in B$ converging to zero in $G_{l}(\vartheta, \varphi, \psi ; x)$ and then we could get that $\lim _{n \rightarrow \infty}\left(K, f_{n}\right)(x)=0$, i.e. the support of $K\left(x-x^{\prime}\right)$ is $G_{l}(\vartheta, \varphi, \psi ; x)$.

In other words, the distributions considered possess the following property. They transform any functions $f(x)$ different from zero in some restricted space-time region $G_{f} \subset R^{4}$ into functions $F(x)=(K, f)(x)$ different from zero only in a some-what larger restricted space-time region $G_{F^{\prime}}=G_{f}+\delta G_{f}$. The region $\delta G_{f}$ is restricted and completely located inside the region $G_{f l}$ such that $x \in G_{f l}$, if $-l^{2} \leqq(x-y)^{2} \leqq l^{2}$ where $y \in G_{f}$. The shape of this ,diffused" region $G_{F}$ should depend only on the behaviour of the function $f(x)$ in the region $G_{f}$.

The physical meaning of the distributions $K\left(x-x^{\prime}\right)$ is the following. Let $\Phi(x)$ be a certain field that appears and then disappears in some restricted space-time region $G_{f}$. Then, due to the "propagator" $K\left(x-x^{\prime}\right)$ this field will affect a certain bounded region with finite fourvolume. This region is completely located inside the region $G_{f l}$. The shape of this region will depend on the "micro-shape" of the pulse of the field $\Phi(x)$. 
The Fourier transforms of the distributions (1.1) with the condition (1.3) can be written in the form (3.8). They are entire analytic functions of the order $1 / 2$ and the type $l$ in the complex plane $p^{2}$, i.e.

$$
\left|\widetilde{K}\left(p^{2}\right)\right|<e^{(l+\varepsilon) \sqrt{\left|p^{2}\right|}}
$$

for any $\varepsilon>0$. Such functions may have only one direction in the complex plane along which they may decrease. Therefore, they can play the role of the cutoff functions in the perturbation series for the Smatrix [10].

The "minimum" space of test functions in this case is the class $B^{l}$ of functions which are analytic in the region $\Gamma_{l} \subset C^{4}$. The point $z \in \Gamma_{l}$ if

$$
-l^{2} \leqq y_{0}^{2}-\mathbf{y}^{2} \leqq l^{2} .
$$

\section{Essentially Non-local Distributions}

Now we consider the sequences $c_{n}$ satisfying the condition (1.4). We restrict ourself to studying only such sequences for which the coefficients $c_{n}$ are real and can be represented as a solution of the following moment problem

$$
c_{n} a_{n}=\int_{0}^{\infty} u^{2 n} d \sigma(u)
$$

where the coefficients $a_{n}$ are given by the formula (3.3) and $\sigma(u)$ is a real monotoneously increasing function. It is possible to demonstrate easily that the coefficients $c_{n}$ in (5.1) always satisfy the condition (1.4).

Making use of (5.1) and performing the calculations as it was done earlier one can obtain for the functional $(K, f)(x)$ the formal representation

where

$$
\begin{aligned}
(K, f)(x) & =\int_{0}^{\infty} d \sigma(u) \int_{\varrho^{2} \leqq 1} d^{4} \varrho f\left(x_{0}+i \underline{\varrho}_{4} u, \mathbf{x}+\varrho u\right) \\
& =\int d^{4} \varrho V\left(\varrho^{2}\right) f\left(x_{0}+i \varrho_{4}, \mathbf{x}+\varrho\right)
\end{aligned}
$$

$$
V\left(\varrho^{2}\right)=\int_{\sqrt{\varrho^{2}}}^{\infty} \frac{d \sigma(u)}{u^{4}} .
$$

$V\left(\varrho^{2}\right)$ differs from zero according to our condition. It is clear generally speaking that this functional is not defined on our space $\mathcal{B}$. It is necessary to coordinate the rate of decrease of the function $V\left(\varrho^{2}\right)$ with the order of the entire functions $f(z) \in 8$ so that the integral (5.2) should be converged. Let us suppose that we have found such a space $B_{v}$ so that the functional (5.2) exists. Obviously this functional is essentially non-local, i.e. it is equivalent to an integral operator whose kernal differs from zero over the whole $\mathrm{x}$-space. 
Nevertheless one can say that the functional (5.2) is characterized by some "elementary length $l$ " in a certain approximate sense. We mean the following. Let a function $f(x)$ differs from zero in some region $G_{f} \subset R^{4}$. Then the functional $(K, f)(x)$ in (5.2) differs from zero in the whole space $R^{4}$. But if the function $V\left(\varrho^{2}\right)$ which is a kernel of the integral operator in (5.2) decreases "rapidly enough" at $\varrho^{2} \rightarrow \infty$ and the region where $V\left(\varrho^{2}\right)$ differs markedly from zero is characterized by a certain effective length $l$, one can say that the distribution $K\left(x-x^{\prime}\right)$ transforms a region $G_{f}$ where $f(x) \neq 0$ into a wider region $G_{f l}$ where the functional $(K, f)(x)$ differs markedly from zero and it is equal approximately to zero in the other points of $R^{4}$. Thus we have in a certain sense the case considered in the section 4 .The question arises what is the "rapidly enough" decreasing of the function $V\left(\varrho^{2}\right)$. How is it possible to formulate the concept of "approximate elementary length $l$ " ?

The "rapidly enough" decreasing is considered usually in physics to be an exponential decreasing, i.e. the function $V\left(\varrho^{2}\right)$ decreases rapidly enough if it satisfies the limiting relation

$$
\lim _{\varrho^{2} \rightarrow \infty} V\left(\varrho^{2}\right) e^{a\left(\sqrt{p^{2}}\right)^{N}}=0
$$

for some $a>0$ and $N>0$. If $N=1$ the decrease is considered to be good enough.

We will study the classes of such functionals for which the function $V\left(\varrho^{2}\right)$ satisfies the condition (5.4) for $N \geqq 1$ and any $a \geqq 0$.

Let us define the space of test functions $B_{N}$. The function is said to belong to the space $\delta_{N}$ if it is the entire analytic function with respect to each of its arguments $z_{j}(j=0,1,2,3)$ of the order less then $\mathrm{N}$, i.e.

$$
\lim _{\left|z_{j}\right| \rightarrow \infty} \frac{\ln \left|f\left(z_{0}, z_{1}, z_{2}, z_{3}\right)\right|}{\left|z_{j}\right|^{N}}=0,(j=0,1,2,3) \text {. }
$$

Let us define the space $\delta_{\infty}=\bigcup_{N} \delta_{N}$ of all entire functions of finite order. It is possible always to find such $N \geqq 1$ that the limiting relation (5.5) is valid.

We will introduce into consideration another space $Z_{b}^{b}[8]$. This space consists of all entire analytic functions $f(z)$ satisfying the inequalities

$$
|f(z)| \leqq C \exp \left\{\sum_{j=0}^{3}\left[-\left|\frac{x_{j}}{\lambda_{j}}\right|^{b}+\left|\frac{y_{j}}{\beta_{j}}\right|^{b}\right]\right\}
$$

for certain positive constants $b, C, \lambda_{j}$ and $\beta_{j}$. The space $Z_{b}^{b}$ is a subspace of $\mathcal{B}_{N}$ for $b \leqq N$. It is not trivial, probided $b>1$.

We will make use of

Theorem. Let $\varphi(x)$ be a piecewise-smooth bounded and absolute integrable function on $R^{4}$ then this function can be approximated uniformaly by $f(x) \in \beta_{N}(N>1)$ with arbitrary accuracy of any $\varepsilon>0$. 
Let us choose such $f(z) \in Z_{b}^{b} \subset \beta_{N}$ which is real for $x \in R^{4}$ and is normalized by the condition

$$
\int d^{4} x f(x)=1
$$

One constructs the sequences of functions

$$
f_{v}\left(x-x^{\prime}\right)=\frac{1}{v^{4}} f\left(\frac{x-x^{\prime}}{v}\right)
$$

where $\nu$ is a real parameter. For each function $\varphi(x)$ satisfying the conditions of the theorem we make to correspond the function

$$
\varphi_{\nu}(x)=\int d^{4} x^{\prime} f_{\nu}\left(x-x^{\prime}\right) \varphi\left(x^{\prime}\right) .
$$

Making use further of usual methods of the mathematical analysis (see e.g. [11]) it is easy to prove that $\varphi_{\nu}(z) \in B_{N}$ and for any $\varepsilon>0$ it is possible to find such $v_{0}$ that for any $v<v_{0}$ the inequality

$$
\left|\varphi(x)-\varphi_{\nu}(x)\right|<\varepsilon
$$

is valid uniformly in all points of continuity of the function $\varphi(x)$ and

$$
\left|\frac{\varphi(x-0)+\varphi(x+0)}{2}-\varphi_{\nu}(x)\right|<\varepsilon
$$

is valid in the points of discontinuity. Thus this theorem is proved.

The functionals $K\left(x-x^{\prime}\right)$ in (5.2) will be defined on the space $B_{x}$ if the corresponding functions $V\left(\varrho^{2}\right)$ in (5.2) decrease not more slowly then an exponent of the order $N$, i.e. it is possible always to find such $A>0$ that

$$
\lim _{\varrho^{2} \rightarrow \infty} V\left(\varrho^{2}\right) e^{A\left(\sqrt{\varrho^{2}}\right)^{N}}=0 .
$$

Let us find the restrictions on the growth of coefficients $c_{n}$ in (5.1). It follows from (5.10) that there exist such $C>0$ and $a>0$ that

$$
V\left(u^{2}\right) \leqq C e^{-a u N} .
$$

Then one can get the following estimate for large $n$

$$
\begin{gathered}
\left|c_{n}\right|=\left|\int_{0}^{\infty} u^{2 n} d \sigma(u)\right|=\left|(2 n+4) \int_{0}^{\infty} d u u^{2 n+3} V\left(u^{2}\right)\right| \leqq \\
\leqq(2 n+4) C \int_{0}^{\infty} d u u^{2 n+3} e^{-a u N}=\frac{C(2 n+4)}{N a^{\frac{2 n+4}{N}}, \Gamma\left(\frac{2 n+4}{N}\right) .}
\end{gathered}
$$

Here we intergated by parts. This relation can be written in another form

$$
\varlimsup_{n \rightarrow \infty} \frac{\left|c_{n}\right|^{\frac{1}{n}}}{n^{\frac{2}{N}}}<\infty .
$$

Only such functionals $K$ will be defined on the space $\mathcal{B}_{\infty}$ for which the 
functions $V\left(\varrho^{2}\right)$ decrease more rapidly than any exponent of finite order, i.e.

$$
\lim _{\varrho^{2} \rightarrow \infty} V\left(\varrho^{2}\right) e^{\left(\sqrt{\varrho^{2}}\right)^{N}}=0
$$

for any N. For example $V\left(\varrho^{2}\right)=\exp \left\{-e^{\varrho^{2}}\right\}$. The condition on $c_{n}$ can be written in the form

$$
\varlimsup_{n \rightarrow \infty} \frac{\left|c_{n}\right|^{\frac{1}{n}}}{n^{\varepsilon}}=0
$$

for any $\varepsilon>0$.

What are properties of functions $V\left(\varrho^{2}\right)$ ? We give

Definition. The function $V\left(\varrho^{2}\right)$ is said to locate approximately in the region $\varrho^{2} \leqq l^{2}$ with accuracy $\varepsilon>0$ if it decreases rapidly enough at $\varrho^{2} \rightarrow \infty$ and

$$
\begin{gathered}
\int d^{4} \varrho V\left(\varrho^{2}\right)=1, \\
\left|\int_{\varrho^{2}<l^{2}} d^{4} \varrho V\left(\varrho^{2}\right)\right|<\varepsilon .
\end{gathered}
$$

The parameter $l$ has a sense of the dimension of the region outside which the function $V\left(\varrho^{2}\right)$ is approximately equal to zero. We introduce the notation

$$
V_{l}\left(\varrho^{2}\right)=\left\{\begin{array}{l}
V\left(\varrho^{2}\right) \text { when } \varrho^{2} \leqq l^{2} \\
0 \text { when } \varrho^{2}>l^{2}
\end{array}\right.
$$

Our problem is to demonstrate that the function $V\left(\varrho^{2}\right)$ in the functional (5.2) can be replaced by the function $V_{l}\left(\varrho^{2}\right)$, i.e. it is possible in a certain approximate sense to consider these functionals as non-local ones with elementary length $l$ (see $\S 4$ ).

First of all we touch on the physical sense of the studied functionals. The functions of a test space describe some distributions of physical quantities, e.g. distributions of a field or a charge or an amplitude of some physical process. These distributions are given in real space-time $R^{4}$ and described by functions which are restricted usually in $R^{4}$. The functional has a sense of a propagator which is connected with the dynamics of a process. We study the space-time properties of the functional $K\left(x-x^{\prime}\right)$ then we have to choose a certain function $\varphi(x)$ which differs from zero in a region $G \subset R^{4}$ and look where the function $\Phi(x)=(K, \varphi)(x)$ will differ from zero in $R^{4}$. But our test function spaces do not contain functions with a finite support. Therefore we have to choose the sequence of functions $\varphi_{v}(x)$ which belong to our space and approximate $\varphi(x)$ with a given accuracy. Let us apply the functional $K\left(x-x^{\prime}\right)$ to the functions $\varphi_{\nu}(x)$ :

$$
\begin{aligned}
\Phi_{v}(x) & =\int\left(K, f_{v}\right)\left(x-x^{\prime}\right) \varphi\left(x^{\prime}\right) d^{4} x^{\prime} \\
& =\int F_{v}\left(x-x^{\prime}\right) \varphi\left(x^{\prime}\right) d^{4} x^{\prime}, \\
F_{v}(x) & =\left(K, f_{v}\right)(x)=\int d^{4} \varrho V\left(\varrho^{2}\right) f_{v}\left(x_{0}+i \varrho_{4}, \mathbf{x}+\underline{\varrho}\right) .
\end{aligned}
$$


Further we will consider the space $3_{N}^{\prime}$. Let $f_{v}(x)$ belong to $Z_{b}^{b}(b<N)$. Obviously we can restrict ourselves to the study of the properties of the functions $F_{v}(x)$. First of all we notice that

$$
\int d^{4} x^{\prime} F_{v}\left(x-x^{\prime}\right)=\int d^{4} \varrho V\left(\varrho^{2}\right)=1
$$

and this integral does not depend on $v$. One estimates the modulus of $F_{\nu}(x)$ :

$$
\begin{aligned}
& \left|F_{\nu}(x)\right|=\left|\int d^{4} \varrho V\left(\varrho^{2}\right) f_{v}\left(x_{0}+i \varrho_{4}, \mathbf{x}+\varrho\right)\right| \leqq \\
& \leqq C \int d^{4} \varrho \frac{1}{v^{4}} \exp \left\{-\frac{\left(\varrho_{4}^{2}+\varrho^{2}\right)^{\frac{N}{2}}}{l^{N}}-\left|\frac{x_{0}}{v \lambda_{0}}\right|^{b}+\left|\frac{\varrho_{4}}{\beta_{0} v}\right|^{b}-\sum_{j=0}^{3}\left|\frac{x_{j}+\varrho_{j}}{v}\right|^{b}\right\} .
\end{aligned}
$$

The estimate can be got by the most simple manner for $N>2$. Making use of the inequality $\left(\varrho_{4}^{2}+\varrho^{2}\right)^{\frac{N}{2}} \geqq\left|\varrho_{4}\right|^{N}+\left|\varrho_{1}\right|^{N}+\left|\varrho_{2}\right|^{N}+\left|\varrho_{3}\right|^{N}$ we obtain

$$
\begin{gathered}
\left|F_{v}(x)\right| \leqq C \exp \left\{-\left|\frac{x_{0}}{v \lambda_{0}}\right|^{b}\right\} \cdot A\left(\frac{l}{v}\right) \cdot B(\mathrm{x}) \\
A\left(\frac{l}{v}\right)=\frac{1}{v} \int_{-\infty}^{\infty} d \varrho_{4} \exp \left\{-\left|\frac{\varrho_{4}}{l}\right|^{N}+\left|\frac{\varrho_{4}}{v \beta_{0}}\right|^{b}\right\} \\
=2 \frac{l}{v} \int_{0}^{\infty} d t \exp \left\{-t^{N}+\left(\frac{l}{v \beta_{0}}\right)^{b} t^{b}\right\}, \\
B(\mathbf{x})=\frac{1}{v^{3}} \int d \varrho \exp \left\{-\sum_{j=1}^{3}\left[\left|\frac{\varrho_{j}}{l}\right|^{N}+\left|\frac{x_{j}+\varrho_{j}}{v \lambda_{j}}\right|^{b}\right\} .\right.
\end{gathered}
$$

The parameter $v$ has a simple sense. If the function $f(x)$ is approximately located in the region $\left|x_{j}\right| \widetilde{z} \lambda_{j}(j=0,1,2,3)$ in the sense of our definition (5.16) the function $f_{v}(x)$ will be approximately located in the region $\left|x_{j}\right| \approx v \lambda_{j}$.

It is easily to see that the function $B(\mathbf{x})$ is approximately located in the region $\left|x_{j}\right| \approx v \lambda_{j}+l$, i.e. the functional can be considered as a quite non-local one with the elementary length $l$.

But the situation is more complicated in the case of a time coordinate. When $l \approx \nu \beta_{0}$ then $A\left(\frac{l}{v}\right) \sim 1$ and the decrease at $\left|x_{0}\right| \rightarrow \infty$ is determined completely by the function $\exp \left\{-\left|\frac{x_{0}}{v \lambda_{0}}\right|^{b}\right\}$. But when $l>v \beta_{0}$ then $A\left(\frac{l}{v}\right) \sim \exp \left\{\right.$ const. $\left.\left(\frac{l}{v}\right)^{-\frac{N b}{N-b}}\right\}$ and the distance on which the function $F_{\nu}(x)$ can be considered negligibly small will be of the order $\left|x_{0}\right| \sim l\left(\frac{l}{\nu}\right)^{\frac{b}{N-\bar{b}}}$. const, i.e. this distance will be large enough for sufficiently small $v$. We get the following picture. If "the elementary length $l$ " does not surpass $v \beta_{0}$ the functional $K$ can be considered as non-local one with the elementary length $l$. But if $v \beta_{0}$ is remarkably smaller then $l$ the region where the function $F_{v}(x)$ can be considered as zero is determined already by the parameter $v$ rather than $l$ 
We want to give the following explanation of the results obtained. Let $\varphi(x)$ describe some physical distribution of a field or a charge, for example. Let the non-locality be characterized by the elementary length $l$. Then in the physical sense the accuracy with which the physical distribution $\varphi(x)$ is given in the space-time $R^{4}$ must not surpass $l$. If it is true we may not distinguish between the distributions $\varphi(x)$ and $\varphi_{\nu}(x)$ where $\nu$ is small enough. Therefore we can assert that the functionals considered are non-local ones with the elementary length $l$.

The analogous estimations can be done for the space $\beta_{\infty}^{\prime}$. In this case the situation is more favourable because the distance where $F_{v}(x)$ can be considered as zero is of the order $\sim l L\left(\frac{l}{v}\right)$ where $L(u)$ is a slowly changing function of a type $\ln u$ or $\ln \ln u$.

The Fourier transforms of the distributions (1.1) with the condition (1.4) are entire analytic functions of any order. If $K \in \mathcal{B}_{N}^{\prime}$ then for $1<N<\infty$

If $K \in \mathcal{B}_{\infty}^{\prime}$ then

$$
\left|\widetilde{K}\left(p^{2}\right)\right| \leqq C \exp \left\{a\left(\sqrt{\left|p^{2}\right|}\right)^{\frac{N}{N-1}}\right\}
$$

$$
\left|\widetilde{K}\left(p^{2}\right)\right| \leqq C \exp \left\{a\left(\sqrt{\left|p^{2}\right|}\right)^{1+\varepsilon}\right\}
$$

for any $\varepsilon>0 . C$ and $a$ are constants.

The "minimum" spaces of test functions in this case are the same spaces $\mathcal{B}_{N}$ and $\mathcal{B}_{\infty}$.

At last let us consider the sequences $c_{n}$ obeying (1.5). The test function space is $\mathcal{B}_{1}$ in this case. The function $V\left(\varrho^{2}\right)$ in (5.2) decreases as linear exponent with respect to $\sqrt{\varrho^{2}}$, i.e. $V\left(\varrho^{2}\right) \sim e^{-a \sqrt{\varrho^{2}}}$ at $\varrho^{2} \rightarrow \infty$. The Fourier transform of this functional is not already an entire function in the complex plane $p^{2}$ because of (1.5). $\widetilde{K}\left(p^{2}\right)$ has some singularities, e.g.

$$
\widetilde{K}\left(p^{2}\right)=\frac{\Lambda^{4}}{\left(p^{2}\right)^{2}+\Lambda^{4}}
$$

where $A$ is a parameter. Usually such functions are chosen as cutoff functions in constructing the perturbation theory for the non-local S-matrix [12]. From our point of view this choice is worst because, firstly, the corresponding function $V\left(\varrho^{2}\right)$ decreases most slowly and, secondly, the singularities of the cutoff function in the complex plane $p^{2}$ destroy the unitarity of the $S$-matrix and we have to introduce some additional rules to save the unitarity.

\section{Conclusion}

We believe that the non-local distributions belonging to the spaces $\mathcal{B}^{\prime}, \mathcal{B}_{\infty}^{\prime}$ and $\mathcal{B}_{N}^{\prime}$ give a reasonable representation for a possible nonlocal nature of the quantum field theory. It would be interesting to 
verify such important theorems of the axiomatic approach as the CPTtheorem, the theorem of local commutativity and so on in the case of the non-local quantum field theory in the sense described above.

The other important problem is the possibility to utilize the nonlocal distributions (1.1) in order to remove the ultraviolet divergences from the perturbation series for the S-matrix. Here the basic problem is to define the product of the distribtions. In the case of the space $3^{l^{\prime}}$ such a product can be defined unambiguously [10].

In conclusion I express my deep gratitude to Professor D. I. BLoKHINTSEV and Doctor I. T. Todonov for discussions.

\section{References}

1. Bogolubov, N. N., and D. V. SHiRkov: Introduction to the theory of quantized fields. Moscow-Leningrad: Gostekhizdat 1957.

2. Streater, R. F., and A. S. Wrghtman: PCT spin and statistics and all that. New York-Amsterdam: W. A. Benjamin, Inc. 1964.

3. Meiman, N. N.: JETP 47, 188 (1964).

4. JAFFE, A. M.: High energy behaviour of local quantum fields. Preprint CLACPUB-249-250 (1967).

5. Titchmarsh, E.: 'Theory of functions. Moscow-Leningrad: Gostekhizdat 1951.

6. Kantorivich, L. B., and G. P. Axilov: Functional analysis in normalized spaces. Moscow: Fiz. math. giz. 1959.

7. Gel'Fand, I. M., and G. E. Shilov: Spaces of test and generalized functions. Vol. II. Moscow: Fiz. Mat. Giz. 1958.

8. - - Usp. Math. Nauk 8, 3 (1953).

9. Martineau, A.: J. Anal. Math. 11, 1 (1963).

10. Efimov, G. V.: Commun. Math. Phys. 5, 42 (1967).

11. Aknieser, N. I.: Lectures about a theory of approximation. Moscow: Nauka 1965.

12. Kirzhniz, D. A.: Usp. Fiz. Nauk 90, 129 (1966). 